\title{
Belphégor
}

\section{Adolfo Fattori, Sparire a se stessi Interrogazioni sull'identità novecentesca}

Crepuscoli del Sé

\section{Linda De Feo}

\section{(2) OpenEdition \\ Journals}

Edizione digitale

URL: http://journals.openedition.org/belphegor/499

DOI: 10.4000/belphegor.499

ISSN: 1499-7185

Editore

LPCM

Notizia bibliografica digitale

Linda De Feo, "Adolfo Fattori, Sparire a se stessi Interrogazioni sull'identità novecentesca », Belphégor [En ligne], 12-1 | 2014, mis en ligne le 12 juin 2014, consulté le 22 septembre 2020. URL : http://

journals.openedition.org/belphegor/499; DOI : https://doi.org/10.4000/belphegor.499

Questo documento è stato generato automaticamente il 22 settembre 2020.

\section{(2) $\Theta \Theta \Theta$}

Belphégor est mis à disposition selon les termes de la Licence Creative Commons Attribution - Pas d'Utilisation Commerciale - Pas de Modification 4.0 International. 


\section{Adolfo Fattori, Sparire a se stessi Interrogazioni sull'identità novecentesca}

Crepuscoli del Sé

Linda De Feo

NOTIZIA

Fattori Adolfo. Sparire a se stessi Interrogazioni sull'identità novecentesca, Ipermedium 2013. 306 p. $€ 18,50$. ISBN: 88-97647-12-6 
1 La casa editrice Ipermedium libri ha recentemente pubblicato il volume Sparire a se stessi. Interrogazioni sull'identità contemporanea di Adolfo Fattori, testo complesso, dotato di rigorosa lucidità analitica e attraversato da preziosissime intuizioni che guidano il lettore lungo le tematiche cruciali dell'interrogarsi novecentesco: l'inesorabile declino del soggetto, la perturbante condizione dello straniamento, il drammatico svincolarsi dall'orizzonte magico-sacrale, il pacificante bisogno della narrazione, l'angosciosa ineludibilità della morte.

È rappresentata la storia del soggetto moderno, che percepisce l'accelerazione del mutamento, compie e trascende i passaggi di stato, le morfogenesi, gli snodi "catastrofici", per dirla con René Thom (Thom, passim), prodottisi nella società capitalistico-borghese.

Adolfo Fattori

Sparire a se stessi

Interrogazioni sull'identità contemparanca

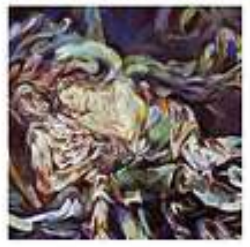

3 Il sociologo napoletano esplora lo statuto contemporaneo di una soggettività alla ricerca dell'edenico incanto perduto, animata dalla nostalgia di un passato mitico e disorientata dal turbinio tecnologico di un futuro imprevedibile, e ne ricostruisce la storia, fino a tratteggiare l'ecologia cognitiva e ambientale realizzata dalla transizione dalle forme storiche dell'immaginario industriale alle forme comunicative della fondazione elettronica.

4 Con lo sguardo rivolto al nesso inestricabile tra processi materiali e modi di rappresentazione, l'autore analizza testi scientifici ed estetici, ripercorrendo il tracciato segnato dalle forme di espressione e di comunicazione, che registrano, interpretano e rilanciano sul piano sia della produzione di immaginario sia del vissuto quotidiano le trasformazioni sortite dai processi di industrializzazione, massificazione, burocratizzazione, secolarizzazione della società. Perlustrando il versante estetico, Fattori ne attraversa le manifestazioni sia alte, elitarie e sofisticate, sia basse, triviali e volgarizzate, liberate dal romanticismo in poi, che riverberano le più potenti pulsioni dei desideri e dei tormenti individuali e collettivi.

5 Lo studioso sottolinea come il romanzo, forma elettiva della narrazione moderna del Sé, si dissolva progressivamente nella ricerca di modalità espressive di epoca in epoca più adatte a interpretare i cambiamenti realizzatisi nella concezione della spaziotemporalità e nella costituzione dell'identità, vale a dire il cinema, la radio, la televisione, la serialità, la cross-medialità, dimensioni in parte nuove e in parte "rimediazioni" (Bolter-Grusin, passim) di quelle classiche.

6 In particolare, con l'intento di tracciare una linea evolutiva tra scrittura e cinema, Fattori si focalizza sulla ristrutturazione percettiva dell'orizzonte spazio-temporale sortita dall'avvento del processo di metropolitanizzazione: descrive lo spezzarsi della categoria del tempo sacro, ciclico, adagiato sul ritmo biologico della natura, nonché il 
prevalere del tempo profano, lineare, determinato dalle scansioni straordinariamente potenti del lavoro industriale, e analizza gli effetti del processo di standardizzazione subito da una serie di traiettorie dello sguardo ad opera di mezzi di trasporto che offrono la possibilità di direzionare i flussi metropolitani, schiudendo varchi allo scorrere episodico delle immagini. Ponendo l'accento sul bisogno di adeguamento alle forme di vita dei modi di rappresentazione, l'autore osserva la progressiva spettacolarizzazione mostrata dalla scrittura prima dell'avvento del cinema, nonché la sua tendenza a intersecare i linguaggi e a riorganizzare i rapporti interattivi tra testo e pubblico sulla dimensione iconica. Ed è sulla capacità di scrittori tuttora presenti nell'enciclopedia immaginativa contemporanea, sensibili ai mutamenti epocali, in grado di interpretare il senso dello slittamento da un immaginario prevalentemente pensato a un immaginario prevalentemente visto, e dei suoi riflessi sull'identità, che Fattori si concentra.

7 Il testo indugia su autori come Edgar Allan Poe, sulle sue teorie riguardanti la filosofia della composizione o la suggestione dell'effetto, sulla sua capacità di rivalutare il lavoro concreto dell'intellettuale, ormai proletarizzato, toccato dalla forza della fabbrica, in modo tale da farlo funzionare secondo la logica dell'industria culturale, caratterizzata dal perfezionamento dei mezzi di produzione e dalla nuova vitalità del ciclo produzione-consumo-produzione. Si sofferma sui rilevanti cambiamenti identitari descritti da Poe, sul ritratto dell'uomo della folla, tipico soggetto sociale metropolitano ottocentesco, già disorientato rispetto agli inarrestabili processi in atto, che, con l'ingresso nel Novecento, troverà compiuta rappresentazione nella narrativa di alcuni scrittori dell'area di lingua tedesca particolarmente sensibili all'accelerazione del tempo e all'avvenuta frammentazione del Sé unitario, destinato a vivere drammaticamente la condizione di estraneità rispetto al mondo. Palesandosi come essere utopico, entità fantasmatica ubiqua, "sempre presente e sempre altrove", "eterno nomade immobile" (Fattori, 2013, pp. 63, 107), questo soggetto, diffuso, fluido, disperso, frammentato, sembra adombrare la soggettività "disseminata" (Caronia, p. 134) nei circuiti di retroazione che collegano il corpo e la sua simulazione nel circolo di integrazione tecno-biologico del World Wide Web.

8 Animando personaggi idealtipici, collocati in "labirinti refrattari a qualsiasi mappatura" (Fattori, 2013, p. 119), che incarnano "gli abitanti della metropoli", "anomici, estremi, irregolari, [...], paradigmatici [...] nella loro apparente eccentricità" (ivi, p. 140), l'opera di Thomas Mann, Robert Musil, Franz Kafka - per citare solo alcuni della folta schiera di letterati su cui Fattori orienta la sua attenzione -, in un vibrante gioco di similarità, mette in scena condizioni di esclusione, orizzonti limbici in cui il tempo e lo spazio sembrano sospesi, ed esprime il disorientamento di un mondo segnato dallo smarrimento delle certezze di tipo ontologico ed epistemologico in un periodo in cui il processo storico sembra essere definito dal rovinare del passato.

Sparire a se stessi percorre il passaggio dalla Weltanschauung della modernità, segnata dalla fede nel progresso lineare e nelle verità assolute, a una concezione della storia intesa non come il precipitato di uno sviluppo necessario, ma come un'accidentata traiettoria, snodata su precipitosi regressi e costruita dalla fallibile verità epistemica, dissolta in un divenire che vanifica ogni fondamento.

10 Sensibilissimo alle tematiche dell'esistenzialità, Fattori, parafrasando il Nietzsche ripreso in Dallo Steinhof anche da Massimo Cacciari, definisce "postumo" (Cacciari, 1980), l'uomo senza qualità di Musil, un uomo che riconosce la costitutività della 
caducità e accetta di esistere in uno "stato di perenne, nobilissima, consapevole precarietà, in cui non c'è stabilità conquistata che non appartenga all'equilibrio instabile della condizione umana" (Piovani, 1989, pp. 158).

11 Contrastando il primato della ragione quale chiave ermeneutica del mondo, nel volume si riflette sulla frattura insanabile con la concezione del tempo che anima le filosofie secolarizzate della storia. Il tempo disarticolato, scardinato, dispiegatosi nei mondi di Kafka o di Mann per raggiungere l'impazzito pluriverso dickiano di Time Out of Joint, tanto frequentato da Fattori, sembra fagocitare il futuro, ricacciarlo nel passato, spezzare sequenze, nella radicale negazione di una teleologia progressiva e nell'affermazione di una concezione della storia che, anziché alla concatenazione degli accadimenti susseguenti, rimanda unicamente all'hic et nunc dell'attimo: ciascuna epoca consiste nella sua stessa esistenza, nel suo essere particolare, così ciascun istante della vita reperisce la pienezza del proprio significato non nel rinvio alla connessione degli avvenimenti, ma nell'Augenblick della propria presenza in cui si coniugano essere e senso.

Il tempo fuori giuntura è il tempo fuor di sesto adombrato secoli prima da un'altra figura che Fattori considera paradigmatica, Amleto, incarnazione del conflitto dell'individuo moderno, introspettivo e solitario, "araldo della morte", "non la morte della tragedia greca, che si risolve in un ritorno alla bellezza della vita, ma [...] la morte della modernità secolarizzata, priva di religione" (Fattori, 2013, p. 253), sradicata da un ordinamento trascendente, che infrange l'incanto del tempo sacro, frantumandolo, producendo disordine. Quella morte in cui "tutte le assenze si verificano vanificandosi", che possiede la "negatività emblematica", conduce alla "conclusiva conoscenza privilegiata" e chiude "il ciclo dall'incognito all'incognito" (Piovani, 1981, p. 122), lasciando affiorare il senso, "irrisolto e irrisolvibile" (Fattori, 2013, p. 286), della fine della vita, anche, e forse soprattutto, quando se ne conosce l'esatta scadenza, come sembra voler mostrare Roy Baty, il re-pipistrello del dickiano Do Androids Dream of Electric Sheep?, diventato il re-folle, Roy Batty, in Blade Runner di Ridley Scott - con lo slittamento di prospettive simboliche che questa trasfigurazione comporta -, tragica metafora della deriva entropica verso cui scivola un'umanità pervicacemente intenta a riformulare se stessa.

Ed è proprio la science fiction, genere letterario particolarmente amato da Fattori, ad essere una modalità espressiva privilegiata degli incubi dell'immaginario operante dalla fine dell'Ottocento fino ai giorni nostri, capace di percepire le vibrazioni dello Zeitgeist novecentesco, di interpretarne le dinamiche e di avvertirne le spinte, di contribuire, e non solo sul piano dell'enciclopedia immaginativa, a ricolonizzare il mondo demagizzato trasformandolo in universo ipertecnologizzato. Progressivamente affrancatasi dai moduli della space opera e dispiegatasi nello "spazio indefinito fra la scienza e l'antiscienza, fra i cercatori della verità ultima attraverso l'assurdo e i profeti di un mondo composto esclusivamente di finzioni" (Hobsbawm, p. 613), la messa in scena della conquista dello spazio, a opera di navicelle cosmiche, è stata sostituita da contributi che, nella seconda metà del secolo XX, costituiscono l'elaborazione di un rispecchiamento che interpreta un senso di rovina diffuso (cfr. Dick, 1955, p. 88) e il prodursi di una nuova articolazione del processo di cambiamento spazio-temporale. Svolgendo il ruolo di narratori metastorici, alcuni autori permettono alla speculative fiction di non mostrarsi più esclusivamente come manifestazione di bisogni, espressione di desideri o esorcizzazione di paure, ma anche come costituzione di forme inedite di 
intelligenza, "ora estetica, ora urbanistica, ora matematica, ora politica, ora religiosa" (Fabozzi-Fattori, p. 319), ora filosofica. Consentendo l'oscillazione tra orizzonte empirico e dimensione segnata dalle ipotesi immaginative, sortendo un effetto di straniamento che, attraverso l'investigazione controfattuale, avvia un processo cognitivo molto fervido, la science fiction si configura come un esercizio espressivo sul tema del possibile, che costituisce il tentativo di comprendere l'esistente, il realizzato, e descrive, con profondità suggestiva e ricchezza di rimandi, realtà che vanno oltre $i$ confini dell'indagine rigorosamente razionale (cfr. Suvin, passim).

Nella produzione fantascientifica viene messo in gioco il corpo, principio primo del senso dell'identità, strumento principe di relazione con il mondo, adombrando la possibilità, diventata sempre meno avveniristica, di realizzare contaminazioni chimeriche, via via più intime, di carne e byte, fino a produrre trasformazioni molecolari destinate alla creazione di vita intelligente non biologica.

L'alterità tecnologica, la presenza aliena emersa nella science fiction, che costituisce sia la reincarnazione, nella letteratura e nelle credenze popolari, degli dei e dei demoni del passato, sia una congettura mitologica, una forma di materializzazione, di proiezione nel vuoto degli archetipi dell'inconscio collettivo (cfr. Jung, pp. 174, 178), contenuto subliminale divenuto visibile, insorgenza dell'inconscio filogenetico, si caratterizza come simbolo dell'irruzione dell'incomprensibile nel territorio della realtà quotidianamente esperita e dallo spazio esterno affiora come alienazione nello spazio interno. Philip K. Dick, autore molto caro a Fattori, "farà del dubbio sullo statuto dell'identità e della realtà la cifra profonda della sua poetica e delle sue trame narrative, fino a paventare [...] l'esistenza di un complotto [...] metafisico" (Fattori, 2013, p. 217), di un complotto cosmico, "inganno radicale prodotto da una qualche impersonale interfaccia deformante collocata fra la coscienza e il reale" (Id., 2001, p. 139).

16 Nel volume si riflette, inoltre, sulla contraddittorietà, a volte solo apparente, tra il razionalizzante disincanto del mondo e il magico amalgama di rigore scientificotecnologico e intuizione trascendente, contaminazione resa spettacolare da alcuni accadimenti, come l'allunaggio, che, nonostante abbia rappresentato un "avvenimento effimero, destinato agli archivi dell'immaginazione scientifica e dell'arroganza tecnologica" (Fattori, 2010, p. 67), in quel lontano 20 luglio del 1969, realizzò compiutamente l'immaginazione fantascientifica, dipanandone i motivi ispiratori, il desiderio di superare un limite ritenuto invalicabile e l'anelito a raggiungere l'ultima meta possibile.

17 Il processo di tecnologizzazione, su cui si fonda l'organizzazione sociale, politica e percettiva, produce un multiverso di realtà virtuali, destruttura gli schemi di comprensione del reale e li sostituisce con un nuovo ordine di realtà. È a tali modelli simulativi che si sono attribuiti i tratti del vissuto, ricostituendo in questo modo un reale ormai quasi completamente atrofizzato, reinventato come finzione (cfr. Baudrillard, 1980, p. 55), che ha smarrito il carattere dell'oggettività, insieme al fondamento ontologico, eroso dall'infinita proiezione di immagini falsificate compiuta dalla fantasia mediatica. Il progressivo, ma inesorabile processo di distacco dalla realtà consueta, nonché di insediamento in un mondo "in cui la più alta funzione del segno è di fare scomparire la realtà e di mascherare al tempo stesso questa sparizione" (Id., 1998, p. 27), trova origine, secondo Jean Baudrillard, proprio nella fantasmagoria, ricordata da Fattori, dell'universo metropolitano delle merci già offerte mediante 
modalità evocative di un reale ideale e assente. L'artificialità, secondo le previsioni baudrillardiane, fagociterà progressivamente il reale, emergendo come "neo-reale" costituito dalla combinazione degli elementi di codice, dei dati numerici della vita, grazie a "una tendenza irreversibile all'astrazione formale degli elementi e delle funzioni [...], al passaggio delle gestualità, dei corpi, degli sforzi, dentro a comandi elettrici o elettronici, alla miniaturizzazione, nel tempo e nello spazio, dei processi la cui scena diventa quella della memoria infinitesimale e dello schermo" (Id., 1987, p. 11).

Il passaggio dalla società dello spettacolo alla cultura dell'informazione, che traduce sull'orizzonte dei processi materiali ciò che popola i territori della science fiction, vanifica ogni possibilità di demistificazione di fronte all'estrema perfezione della tecnologia contemporanea: gli ologrammi, le raffigurazioni tridimensionali, gli ambienti virtuali, emanazioni dei codici digitali che li generano, allontanano il soggetto dal proprio referente originario. Non c'è crisi della realtà, anzi ci sarà sempre più realtà, prodotta e riprodotta mediante la più spaesante delle simulazioni.

Nelle pagine di questo illuminante testo si intravede tuttavia la disperata volontà di affermazione di un'esile possibilità di riscatto, grazie a un ancoraggio, quello offerto dall'universo simbolico della narrativa, nonostante l'ineludibile condizione di disorientamento sia insita nella scrittura stessa, che evoca l'essere nella modalità dell'assenza, nella dimensione della mancanza, che rappresenta sull'orizzonte dello smaterializzato.

L'uomo che abita un altrove spaziale e temporale, una realtà progressivamente meno solida, conserva gli strumenti dell'osservazione e della descrizione, rendendo il testo narrante fondamentale per la sopravvivenza: selezionando gli eventi e ordinandoli in sequenze significative, illuminando la complessità delle molteplici connessioni relazionali e attribuendo un senso a sezioni finite dell'insensata infinità dell'accadere, la narrazione, sembra suggerirci Fattori, ha da sempre costituito una dimora per chi, in modi diversi, si sente nomade, e, a titolo differente, conosce la condizione dello spaesamento, anche quello sortito dalla tecnologia, aiutando così a vivere nel perturbante stato dell'Unheimliches, dell'essere senza casa o patria, stato ormai diffuso del sentire nell'attuale media landscape, a ritrovare se stessi nell'irriconoscibilità di un luogo alieno, cercando di resistere al tragico gioco di dissolvenze che sfuma sull'orizzonte storico l'avvicendarsi delle identità trasformate, e a recuperare l'irriducibilità del proprio essere situati nel mondo.

\section{BIBLIOGRAFIA}

A. Abruzzese (a cura di), Materiali di sociologia della letteratura, Napoli, E. DI. SU., 1992.

Jean Baudrillard, Simulacri e fantascienza, in L. Russo (a cura di), La fantascienza e la critica, cit.

Jean Baudrillard, L'autre par lui-méme. Habilitation, 1987, trad. it. di M. T. Carbone, L'altro visto da sé, Genova, Costa \& Nolan, 1987. 
Jean Baudrillard, Illusion, désillusion esthétiques, 1997, trad. it. di L. Guarino, Estetica della disillusione, in V. Valentini (a cura di), Allo specchio, cit.

J. D. Bolter-R. Grusin, Remediation. Understanding New Media, 1999, trad. it. di B. Gennato, Remediation. Competizione e integrazione tra media vecchi e nuovi, a cura di A. Marinelli, Milano, Guerini e Associati, 2003.

Massimo Cacciari, Dallo Steinhof, Prospettive viennesi del primo Novecento, Milano, Adelphi, 1980.

Antonio Caronia, Il corpo virtuale. Dal corpo robotizzato al corpo disseminato nelle reti, Padova, Muzzio, 1996.

Philip K. Dick, Time Out of Joint, 1959, trad. it. di A. Martini, Tempo fuor di sesto, Roma, Fanucci, 2003.

Philip K. Dick, Do Androids Dream of Electric Sheep?, 1968, trad. it. di R. Duranti, Ma gli androidi sognano pecore elettriche?, Roma, Fanucci, 2000.

Philip K. Dick, The Shifting Realities of Philip K. Dick: Selected Literary and Philosophical Writings, (ed. by Lawrence Sutin), 1995, trad. it. di G. Pannofino, Mutazioni. Scritti inediti, filosofici, autobiografici e letterari, Milano, Feltrinelli, 1997.

Philip K. Dick, Pessimism in Science Fiction, 1955, in Id., The Shifting Realities of Philip K. Dick: Selected Literary and Philosophical Writings, cit., trad. it. Pessimismo e fantascienza, in Id., Mutazioni. Scritti inediti, filosofici, autobiografici e letterari, cit.

Antonio Fabozzi, Adolfo Fattori, Fantascienza, in A. Abruzzese (a cura di), Materiali di sociologia della letteratura, cit.

Adolfo Fattori, Memorie dal futuro. Spazio, tempo, identità nella science fiction, Napoli, Ipermedium libri, 2001.

Adolfo Fattori, Cronache del tempo veloce. Immaginario e Novecento, Napoli, Liguori, 2010.

Adolfo Fattori, Sparire a se stessi. Interrogazioni sull'identità contemporanea, Napoli, Ipermedium libri, 2013.

E. J. Hobsbawm, Age of Extremes - The Short Twentieth Century 1914-1991, London, Michael Joseph, 1994, trad. it. di B. Lotti, Il secolo breve, Rizzoli, Milano, 1995.

C. G. Jung, Ein moderner Mythus. Von Dingen, die am Himmel gesehen werden, 1958, in Id., Zivilisation im Übergang, 1974, trad. it. di S. Daniele, Un mito moderno: le cose che si vedono in cielo, in Dopo la catastrofe, Torino, Bollati Boringhieri, 1998.

G. Lissa (a cura di), Posizioni e trasposizioni etiche, Napoli, Morano, 1989.

P. Piovani, Etica, in Enciclopedia del Novecento, Roma, 1977, ora in G. Lissa (a cura di), Posizioni e trasposizioni etiche, cit.

P. Piovani, Oggettivazione etica e assenzialismo, a cura di F. Tessitore, Napoli, Morano, 1981.

L. Russo (a cura di), La fantascienza e la critica, Milano, Feltrinelli, 1980.

D. Suvin, Metamorphoses of Science-Fiction. On the Poetics and History of a Literary Genre, 1979, trad. it. di L. Guerra, Le metamorfosi della fantascienza. Poetica e storia di un genere letterario, Bologna, il Mulino, 1985.

R. Thom, Stabilité structurelle et morphogénèse. Essai d'une théorie générale des modelès, 1972, trad. it. di A. Pedrini, Stabilità strutturale e morfogenesi. Saggio di una teoria generale dei modelli, Torino, Einaudi, 1980. 
V. Valentini (a cura di), Allo specchio, Roma, Lithos, 1998. 